- Stephen C. Levinson

MAX PLANCK INSTITUTE FOR PSYCHOLINGUISTICS

\title{
Language and Cognition: The Cognitive Consequences of Spatial Description in Guugu Yimithirr
}

This article explores the relation between language and cognition by examining the case of "absolute" (cardinal direction) spatial description in the Australian aboriginal language Guugu Yimithirr. This kind of spatial description is incongruent with the "relative" (e.8., left/right/front/back) spatial description familiar in European languages. Building on Haviland's 1993 analysis of Guugu Yimithirr directionals in speech and gesture, a series of informal experiments were deoeloped. It is shown that Guugu Yimithirr speakers predominantly code for nonverbal memory in "absolute" concepts congruent with their language, while a comparative sample of Dutch speakers do so in "relative" concepts. Much anecdotal evidence also supports this. The conclusion is that Whorfian effects may in fact be demonstrable in the spatial domain.

\section{Language and Cognition}

Does the language you speak have any effect on the way you think? The presumption in recent years has been no. Most important cognitive processes are deemed to have a universal organization, and languages themselves have been shown to conform to many universal principles. The idea-associated with the names of Humboldt, Whorf, and Sapir-that particular expressions in particular languages might induce particular ways of thinking about varied subject matter has few modern proponents. 
But in fact it is self-evident that certain linguistic distinctions force attention to certain properties of the world. In order to describe a sequence of overlapping events in English, one simply has to attend to the temporal relations between the events, as required by the tense and aspect systems of the language, systems missing in part or whole from certain languages, for example, Hebrew and Malay. In contrast some languages require coding of, for example, whether referents are visible to the speaker (Kwakwala), observed directly or only heard by report (Turkish), or oriented in particular ways (Tzeltal). Studies of language acquisition have highlighted just how profoundly different such distinctions can be and how early sensitivity to them is acquired, even before their production is mastered (Bowerman 1996; Bowerman and Choi in press; Choi and Bowerman 1991).

Drawing attention to this, Slobin (1996) has suggested that we must concede that a specific language, with its associated grammatical and semantic categories, requires a specific mode of thought at least during on-line speech production. The question that then arises is: are such cognitive effects restricted to "thinking for speaking," or do they extend beyond, to general processes of attention, memory, and inference? Slobin (1996) and Levelt (1989) are agnostic but perhaps incline to the view that thinking for speaking will have little demonstrable effect upon thinking in general. Lucy (1992) thinks otherwise. (See also Gumperz and Levinson 1996a for a compendium of recent views.) This article explores this question with special attention to the language of spatial description in an Australian language (see also Haviland 1993).

\section{Absolute versus Relative Modes of Spatial Description}

It has been proposed by psychologists and linguists that the language for describing space mirrors fairly directly universal ways of thinking about space. Thus Piaget demonstrated how the linguistic expressions for many spatial distinctions must await the development of the corresponding conceptual categories during the maturation of the child before they can be used properly. More recent studies of linguistic development in the child seemed to support this picture, with the acquisition of linguistic terms following an order presumed to be dictated by universal stages in development of the underlying concepts (Clark 1973; Johnston and Slobin 1978). Meanwhile, cognitive psychologists had presumed that the sorts of concepts expressed in the prepositions of the familiar European languages also formed the universal basis for naive concepts of space (Clark 1973; Miller and Johnson-Laird 1976). The picture that emerged was that we are innately, or environmentally, predisposed to conceive of space relatively, that is, egocentrically, with no fixed angles, relying on relational concepts such as "in front of," "behind," "to the left of," and so forth, to describe the relative dispositions of objects from a particular point of view.

Work by the Cognitive Anthropology Research Group at the Max Planck Institute in Nijmegen has now drawn attention to the fact that there are many languages that require their speakers to make reference to absolute angles, angles fixed once and for all, such as north, south, east, and west in 
English (see, e.g., Brown and Levinson 1993). Clearly, English speakers have available such expressions too, but it has been assumed, surely correctly, that these are not central to our naive perception and conception of space. Linguistically, we do without them most of the time, utilizing a rich set of prepositions and prepositional phrases (such as across, in front of, opposite, beneath, on the side of, etc.) to describe the locations of objects. For distinguishing sides of our visual field or describing rotation and indeed many other things, we make central use of notions of left and right.

But there are languages that encode very few "prepositional" notions, do not use left and right in an extended spatial sense, and indeed require the conception of spatial relations in a fundamentally nonrelative manner. ${ }^{1}$ It is one of these, Guugu Yimithirr, that is the subject of this study. In Guugu Yimithirr (henceforth GY) nearly all spatial descriptions involve essential reference to something like our cardinal directions. ${ }^{2}$ In GY, in order to describe someone as standing in front of the tree, one says something equivalent (as appropriate) to "George is just north of the tree." To tell someone to take the next left turn, one might say "Go north"; to ask someone to move over a bit, "Move a bit east"; to instruct a carpenter to make a door jamb vertical, "Move it a little north"; to tell someone where you left your tobacco, "I left it on the southern edge of the western table in your house"; to ask someone to turn off the camping gas stove, "Turn the knob west"; and so on.

So thoroughgoing is the use of cardinal directions in GY that, just as we think of a picture as containing virtual space, so that we describe an elephant as behind a tree in a children's book (based on apparent occlusion), so GY speakers think about it as an oriented virtual space: if I am looking at the book facing north, then the elephant is north of the tree, and if I want you to skip ahead in the book, I'll ask you to go further east (because pages are flipped from east to west).

Such a language makes elaborate and detailed reference to an absolute set of angles: absolute in the sense that they do not depend on the angle of the human frame (unlike left and right) nor, essentially, on the speaker's view point (unlike in front of the tree), although ego's position may optionally be used as a reference point (as in north of $m e$ ). If you describe the layout of a room in an absolute system, the description does not vary whether you look through the window or the door: for example, the lamp is north of the sofa, with the table to the west. In a system based on relative angles, in contrast, the lamp may be behind the sofa with the table to the left when viewed from the door, but when viewed from the window the sofa may be behind the lamp with the table to the right. Thus we shall oppose a relatioe system of conception and description to an absolute one. Anyone who doubts the fundamental difference between these two kinds of systems should try to devise a general instruction, purely in absolute terms, about how to lay the table in genteel fashion (e.g., forks to the left, knives to the right).

Guugu Yimithirr is a language more or less completely absolute in spatial description. The argument advanced here will be that, in order to speak GY, it is necessary to carry out a specialized kind of background computation of orientation and direction. Further, these computations must be carried 
out well in advance of (indeed independently of) speaking, and the results must be memorized, be available for inference and other psychological process, and in general pervade many aspects of cognition. Thus the fact that absolute directional information is a fundamental prerequisite for speaking GY must have pervasive psychological implications.

The argument is made in two ways. The first line of argument consists of qualitative information (in the way of natural records of interaction and anecdote) about the nature of the linguistic distinctions and their mode of application. Various kinds of linguistic performance have been induced by setting systematic tasks of spatial description. It is argued that, in order for these linguistic performances to be possible, psychological processes of special kinds must be involved. ${ }^{3}$ The information here supplements work by Haviland (1979, 1993, n.d.[a]). The second line of argument is based on informal experiments that attempt to assess psychological variables directly, independently of language. ${ }^{4}$

\section{Guugu Yimithirr Communication about Space}

\section{Guugu Yimithirr Spatial Description: The Linguistic Resources}

Like most Australian languages, Guugu Yimithirr makes essential use of terms for cardinal directions. ${ }^{5}$ There are four root expressions, as in our western compass points, but the roots label "edges" (i.e., quadrants) rather than points (despite which we shall from time to time gloss them as "north" etc.) and are skewed slightly clockwise from our magnetic or grid directions (Haviland 1979, n.d.[a]). The linguistic details are not essential to this article, and I give the briefest account here, largely derived from work by Haviland $(1979,1993$, n.d.[a]). The cardinal direction roots are:

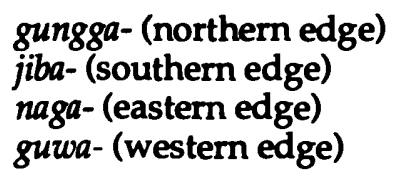

Unlike the privileged position that Western civilization confers on north (on account of the magnetic compass and our map making tradition), there is no obvious priority to any one direction or axis in GY. It is interesting, for example, that in the "brother-in-law language" (an alternate vocabulary used when talking in the presence of taboo relatives), only two of the four roots are thought necessary to be replaced, but since they are the terms for south and west, no clear pattern of priority emerges. ${ }^{6}$

The directional roots appear with various morphological derivations, of which the most important are as follows. (I follow Haviland's 1993 analysis, which is by no means self-evident but, I think, is correct; glosses approximate only.) 


\section{jibaarr 'to/at the $\mathbf{S}^{\prime}$}

naga 'to/at the $\mathrm{E}^{\prime}$

guwa 'to/at the W'

These are the simplest, unmarked forms, used for example in route directions of the form "turn west," and have a locative or allative interpretation.? Another frequently used set contrasts by implying some kind of focus on the end point of a journey or trajectory:

R-form: (end-point focus)

gunggarra 'to/at a point in the $\mathrm{N}^{\prime}$

jibarra 'to/at a point in the $S^{\prime}$

nagaar 'to/at a point in the $E^{\prime}$

guwaar 'to/at a point in the $W$ '

These are then the terms naturally used if one is specifying a destination. (Indeed, ngayu gunggarra thadaara 'I am going north' may already be sufficient to unequivocally specify that I am going, for example, to the beach.) The third frequently used set puts some kind of focus or emphasis on the trajectory or vector itself, as in motion through a point; these terms are thus naturally used to describe a general direction of heading, or an alignment.

\section{L-form: (vector) \\ gunggaalu 'northwards' \\ jibaalu 'southwards' \\ nagaalu 'eastwards' \\ guwaalu 'westwards'}

When specifying motion from a direction, there are corresponding forms with different ablative inflections. When specifying small movements or small distances, reduplications do the job. There are also special derivatives for specifying northern, southern, eastern, and western edges (e.g., the east side of a house, or the eastern flank of a mountain range). Altogether, there are at least 12 derivational suffixes, together with a number of case inflections (e.g., the comitative case), yielding for the four cardinal roots something like $\mathbf{5 0}$ distinct forms with distinct meanings."

Since Guugu Yimithirr does not employ terms for left and right in a spatial sense and does not directly encode notions of relative location such as English in front of, behind, and at the side of, it is essential to use these cardinal direction terms for almost every description of location on the horizontal.' It is also customary to specify direction when using verbs of motion.

The interpretation of these cardinal expressions is not as straightforward as might at first seem. Although these terms refer to fixed angles, the origin point from where the angle is subtended can be of various kinds. For example, "I went north" may be interpreted as "I went north from here," "I went north from there" (place given by marrative), or just "I headed in a northerly direction" (see Haviland 1996). 
Guugu Yimithirr is a language under rapid change, and the present Hopevale Community where it is most often (but not exclusively) spoken is a sociolinguistically complex one, with a long mission history and a rapidly expanding population. ${ }^{10}$ No adequate study of the varieties of the modern language has been done. ${ }^{11}$ All speakers of GY are also speakers of English, and for many Hopevale residents English is now the predominant language. The generalizations in this study are based on data gathered almost exclusively from men over 40 , indeed, mostly in the age bracket 55-75, who would normally speak Guugu Yimithirr amongst themselves in preference to English and who have spent their lives in close association with each other and the land of Hopevale aboriginal mission. Reference to "GY speakers" and the like in this article should be interpreted in that restrictive fashion. I simply do not know to what extent these results would generalize to a larger sample, inclusive of both sexes, but there is no doubt that the picture would be much less straightforward. ${ }^{12}$

\section{The Communicative Use of Cardinal Direction Information}

There has been extensive study of the use of directional terms in GY verbal interaction and story-telling (see Haviland 1993, 1996, n.d.[a]; also see Levinson 1987a, 1987b, n.d.[c]). These studies show that directional terms have a high frequency of use (Haviland estimates 1 word in 10) and also that directions are specified with a consistency and accuracy that allows them to play an essential role in reference identification and tracking. (For example, to report a conversation, the interlocutors may be identified as the one to the north and the one to the south.)

Haviland (1993, n.d.[a]) has also shown in detail through the study of filmed interaction how this linguistic system is supplemented by gestural specification, where directional gestures have great accuracy, although their interpretation requires understanding of the point of view from which the gesture is made (Haviland 1996). The fact that gesture is deeply integrated into the system of directional reference is fundamentally important to an understanding of the cognitive background to the system. It demonstrates clearly that it is not simply a linguistic system but a broader communicative one.

In addition to this body of natural spoken interaction,,$^{13}$ as part of the current study a corpus of spatially directed talk was systematically collected from older GY speakers. A battery of elicitation techniques was used, as developed by the Max Planck Cognitive Anthropology Group as part of a cross-linguistic study of spatial description and conception involving over a dozen non-Indo-European cultures. ${ }^{14}$ Informants were asked to perform a series of 11 interactive tasks, or "games." For example, in one of these tasks, a "director" had to describe a route (marked with a cord) through a model town, so that another informant (the "matcher") could emulate the route on an identical, but screened-off, model. This essentially involved giving precise route and location descriptions. In another task, the director had to describe the position and stance of an articulated wooden man, so that the matcher could emulate the body positions on another 
identical figurine. In a third task, the director had to describe the relative locations of a set of farm animals so that the matcher could emulate the arrangement. And so on. ${ }^{15}$

These tasks push the resources for spatial description of any language to the limit, and it was interesting to note the almost total reliance of our GY speakers on the directional system. Rotation, angular direction, side or edge location, relative distance, and so on were all specified essentially through cardinal direction terms. Even in the task involving the figurine, where terms for left and right body parts might have been used (the lexicon includes nominals for "left hand" and "right hand" in a purely body-part sense), specification was exclusively in terms of cardinal locations ("hand on the western side," etc.). As a result, the orientation of models or assemblages was always identically matched in absolute terms (e.g., the model man not only pointed with his left hand but pointed west with his left hand), regardless of the relative positions of the director and the matcher.

\section{The Hypothesis of Nonlinguistic Consequences}

Against this background it was decided to investigate the cognitive consequences of operating a communicative system of this sort, both verbal and gestural. The hypothesis was that the cognitive consequences would be far-reaching. Although most perceptual properties are no doubt universal, given by the interaction between the structure of the perceptual organs and that of the relevant perceptual field, the further processing of perceptual information may bring it into line with what is required for the communicative system. For example, if I am to be able to describe a scene at some later point, I must remember the properties required for accurate linguistic description in my particular language. Thus to describe an arrangement of model farm animals in English, I must remember, for example, that the pig was in front of the cow, with the horse, say, to my left or its right. But to do so in GY I must remember the cardinal directions of each animal, or at least be able to reconstruct them. ${ }^{16}$ Thus although the perceptual information available to GY and English speakers may be identical, the coding for memory must, it seems, be distinct. ${ }^{17}$

The hypothesis is that GY speakers must take primary visual perceptions and code them together with cardinal direction information for memory. They must do this all of the time, since one never knows in advance what memorized facts one may wish to describe. Inference on such coded memories should make use of this cardinal direction information. In short, one expects that GY speakers might behave differently in memory tasks, whether recognition or recall, and in inferential tasks, than speakers of a language with "relative" spatial description.

\section{The Essential Prerequisite: Orientation and Mental Maps}

GY speakers invariably seem to know, day and night, familiar or unfamiliar location, whether sitting still or traveling in a vehicle, where the cardinal directions lie. At night in a vehicle on a winding road, they can 
point accurately at random intervals to cardinal points. For example, en route to Cairns for a land rights meeting, a group of men on a bus before dawn repeatedly gestured the boundaries of traditional territories held by various lineagegroups, saying, for example, "It runyarrba, this way," even though the bus was passing through a very rough and circuitous stretch of road.

This orientational surety is considered trivial and is general, I have the impression, throughout the speech community, despite thesociolinguistic diversity. There are many details of everyday interaction that exploit this surety. Quite typical, for example, is the following advice given to me in English:

"They have plenty of fish fillet in the store."

"T've never seen it; where?"

"On this side" [gesturing] "in the frozen food container, far end this side."

We were standing in a hospital 45 kilometers from the said store. I was expected to note that the gesture was to the northeast, so that next time I was in the store I would look in the northeast corner.

But in order to speak GY, more than a sense for immediate direction is required. In order to say, for example, that George was standing in front of the post office in Cooktown, it will be necessary to remember (or be able to calculate) the orientation of the building and George's location relative to that, so that we can say in effect, "George was standing east of the post office." Similarly, if he was at some point A heading for B, a proper description will include the quadrant containing the directional angle between $A$ and $B$.

To achieve such descriptions one will need to know, or be able to recover, the angles subtended between any two places. In that sense a mental map of one's world with accurate absolute angles must be accessible. One place in that map that will be especially, but not exclusively, important will be the location of speaking, since perhaps the majority of cardinal expressions have a deictic interpretation ("north of here"). As the speaker moves, his angular position vis-à-vis other places will change. In that sense, it will be essential to dead reckon one's current location, in order to know what angles the current position subtends to places one might wish to refer to.

How accurate is the sense of direction? In particular, how accurate is the dead reckoning on which the use of GY directionals would seem to depend? ${ }^{18}$ An attempt was made to systematically investigate this, by getting people to point at distant invisible locations from different transitory locations, for example, on stops while traveling or camping. On various trips to the bush or at halts on other kinds of journeys, ten men aged 35 to over 70 were asked on various occasions to estimate directions of named locations beyond the range of vision. The locations were chosen because they had names familiar through local history or associations, even though not all informants had visited all of them. The sites were variously mountains, river mouths or crossings, islands, cattle stations, promontories, old mission sites, and the like. The distances of these locations ranged from a few kilometers up to several hundred, as the crow flies (and up to about 350 kilometers as the routes actually go). The method consisted of halting 
at some spot with restricted visibility (e.g., among trees), asking the men to point to a series of locations, if possible by picking out a landmark in the direct line of sight, so that the investigator could sight through a prismatic compass. It was made plain that an accurate rather than an instant response was being asked for, but in most cases the response was within a couple of seconds (some exceptions noted below), often an immediate gesture. Later the readings were compared to the most detailed survey maps available, the location at which the questioning took place identified (where necessary by triangulation from landmarks and by odometer or pedometer readings), the angles between that place and the pointed-out locations measured, $6^{\circ}$ allowance made for the difference between magnetic and grid (map) north, and the subjects' errors calculated. ${ }^{19}$ In some of the most interesting situations, being remote and relatively inaccessible, I was unable later to accurately locate my own position and had to discard the measurements.

There are many possible sources of investigator error here due, for example, to misidentifying the sightline, compass fluctuation, confusion or uncertainty over the names of places, to misidentifying the target location (due to mistaken identification of unmapped locations), and so forth. Informant patience was also a limiting factor. So enough readings were taken to even out investigator errors.

The results are shown in Table 1. Over a total of 120 trials from bush locations or stops on bush roads, the average error was $13.9^{\circ}$, or less than 4 percent. Including estimates from base camp (Hopevale), there were a total of 160 trials, with an average error of $13.6^{\circ}$, a median error of $11^{\circ}$, and a fairly even distribution of errors in both directions, clockwise (67) and anticlockwise (79) from north (the remainder being zero errors). Forty-five errors (28 percent) were of $5^{\circ}$ or under.

Table 1

Mean corrected error of Hopevale subjects' estimates of direction.

\begin{tabular}{|c|c|c|c|c|c|}
\hline & $\mathbf{N}$ & Degrees & Percent & Median & SD \\
\hline $\begin{array}{l}\text { From nonbase locations } \\
\text { Including base and }\end{array}$ & 120 & 13.9 & 3.9 & 11.0 & 11.3 \\
\hline hypothetical locations ${ }^{b}$ & 160 & 13.5 & 3.8 & 11.0 & 11.2 \\
\hline From base locations only & 40 & 12.6 & 3.5 & 9.5 & 11.0 \\
\hline From hypothetical locations & 6 & 19.0 & 5.2 & - & 16.0 \\
\hline Ten worst estimates & 10 & 38.8 & 10.8 & 37.5 & - \\
\hline Ten best estimates & 10 & 0.2 & 0.1 & 0.0 & - \\
\hline
\end{tabular}

Note: I have retained a conventional statistical presentation for comparison with Lewis's results in Table 2. But there is a sounder method for the statistical treatment of circular estimates (Batschelet 1981). I have recalculated my data by that method for the purposes of a comparative study which can be found in Levinson 1996b.

a Base here means Hopevale.

${ }^{b}$ Hypothetical locations are actual distant places where the subject was asked to imagine he was located before pointing to other places. 
Table 2

Mean corrected error of Western Desert peoples' estimates of direction (Lewis 1976).

\begin{tabular}{|c|c|c|c|c|c|}
\hline & $\mathbf{N}$ & Degrees & Percent & Median & SD \\
\hline From nonbase locations & 34 & 13.71 & 3.8 & 7.0 & 16.48 \\
\hline From hypothetical locations ${ }^{\mathrm{a}}$ & - & - & - & - & - \\
\hline Ten worst estimates & 10 & 35.8 & 9.9 & 27.0 & - \\
\hline Ten best estimates & 10 & 1.3 & - & 0.5 & - \\
\hline
\end{tabular}

Note: The worst estimate was $67^{\circ}$ out; the best estimate had zero error. Lewis correlated degree of error with angular bearing to axis of travel: places off to starboard or port, as it were, were harder to point to, because they required accurate allowance for speed and therefore distance of travel. (They are also not places naturally being thought about, unlike places on the axis of travel.) Given that travel in GY territory is nearly always circuitous, it is harder to test this source of error.

abilities to estimate direction from hypothetic locations were tested, but the figures were not given.

Given the varied nature of the locations where readings were taken (true bush to roadside), the different speed of travel (foot, vehicle on good gravel road vs. bush track), the approximate nature of some of the readings due to impatience, lack of time, impending dusk, and so forth, the great distance of some of the locations pointed to, and in many cases their relative unfamiliarity, these are very impressive results (especially compared to the performance of Europeans). ${ }^{20}$ By way of contrast, accuracy improved slightly by about $1^{\circ}$ average error when persons were asked locations from base at Hopevale and decreased to about $20^{\circ}$ average error when asked at Hopevale to estimate angles subtended from a distant place A ("hypothetical location") to another one B.

Individuals' abilities vary, as indeed does their experience of the various locations. The best subjects could achieve better averages than this: for example, two of the best could approach $10^{\circ}$ average error, although even the best estimators made more substantial errors from time to time. Bearing in mind that some of the locations pointed to are very remote and scarcely visited, this sort of accuracy displays a fairly remarkable sense of orientation vis-d-vis other locations from a random site. This degree of accuracy probably considerably exceeds one's ability to communicate it through gesture, as it seems hard to make adequate allowance for parallax when estimating exactly where another person is pointing.

The figures may be usefully compared to the only other comparable data from Australia, those collected by Lewis (1976) in desert conditions from Western Desert peoples. Lewis's study was a direct inspiration for this one. Over two fieldtrips, he took 34 measurements during desert travel by vehicle, from five Western Desert aboriginal men of different tribes. Table 2 extrapolates from his results.

The figures are interestingly comparable, despite the very different conditions under which they were collected. In Lewis's experiment, the distances of the locations ranged from 10 to 670 kilometers but were mostly 
around 300 kilometers. Our GY experiment had the ranges 7 to 350 kilometers but were mostly in the 50-100 kilometer range. He was traveling off-road over great distances, while we were mostly on or at least only a few kilometers from dirt roads or bush tracks. On the other hand, his subjects often had the benefit of vistas over vast terrain, while GY terrain is very much rougher, and except along the coast, visual sighting is cut by dense bush, dunes, and mountain ranges. Above all in GY territory, it is almost never possible to travel in a straight line, as the route must deviate constantly around bogs, deep rivers, mountains, sand dunes, rain forest or impenetrable scrub, mangrove swamp, or, if on foot, around snake-infested grassland.

The pattern of GY errors is worth remarking on. On some occasions, there was a clear rotation of many points by up to $20^{\circ}$, while maintaining relative angles. Local points were sometimes especially prone to error; even the central mission site ("home base" for most informants) was often erroneously located. This at first surprising result is, of course, occasioned by the relatively great change of angle of local points (compared to distant points) experienced during our relatively short bush trips and shows that, while the consultants maintain a firm conceptual framework of absolute locations, their dead-reckoning skills are not always as good as their orientational ones. The ten largest errors in the larger sample (including estimations from home base at Hopevale) varied between 51 and $33^{\circ}$ from the true direction. The ten locations pointed to varied in distance from 12 to 114 kilometers in a straight line from the place of the test, but such distances have little bearing on the length of route to reach the destination; for example, the shortest distance here, 12 kilometers, involves about 60 kilometers' travel over dirt road and bush track. Six of these largest errors surprisingly involve base (Hopevale) as origo or destination, and one of the explanations must be that the road to Hopevale from Cooktown describes a circuitous half circle. (Three of the largest errors were from points on this road reached by fast travel, which might disturb a sense of angular orientation.)

In contrast, the ten most accurate estimates showed at most 1 measured degree of error, with locations 8-270 kilometers away. Six of these, with no measurable error, were estimations of location of the old mission site at Cape Bedford from various locations within 30 kilometers. Here accuracy is clearly linked to proximity and familiarity, for the old mission site at Cape Bedford was a location from which people walked all over the reservation.1

What these results show is that GY speakers maintain a mental map of a large terrain, say 300 kilometers by 150 kilometers in extent, and can compute their own location within it and the angles between that location and any others, more or less at the speed of conversational response.

What is this ability based on? Clearly, a constant sense of orientation (the perceived triviality) is one ingredient. The basis for this is still unclear to me. Systematic use of the sun as a compass is by far the most likely basic check on inertial navigation. In the southern winter, the sun lies discernibly (say $12^{\circ}$ ) north at midday at this latitude of $15^{\circ}$ and sets and rises north of west and east. Use of a sun compass of course requires constant correction for time of day and progress of season. But three men independently volunteered anecdotes about how, when traveling far afield (Melbourne, 
Brisbane, or New Guinea), they became convinced the sun was rising other than in the east (Haviland also collected anecdotes of this kind), while another seemed to claim that this was actually the case in distant places. This suggests that at least on some occasions primary reliance is placed on other clues including sheer memory of angular rotation. Bush lore includes ways of discerning orientation from the relative brightness of sides of trunks of particular kinds of trees, from the reliable orientation of certain kinds of termite mound, from the prevailing wind directions at particular seasons, the nightly swarm of bats, the migratory flight of bird species, the rising and setting points of particular stars, the northeasterly alignment of sand dunes in the coastal area, the prevailing currents around particular stretches of the coast, and so on, and presumably all this kind of information is constantly if unconsciously monitored.2

Multiple sources of information must thus be used to constantly update a mental map of one's own current location in a large territory. But to achieve this, other kinds of information are necessary too. The other ingredients include abilities to estimate distance traveled on each leg or angle of a journey. On some of the few occasions on which I obtained delayed responses, informants would give a protocol of a mental dead reckoning of the form, for example, "We came a little bit west, then went that long way straight north along the dune, then had to go west again along the river bank, crossed it, and then could go east again; so where we started from would be about there." The pattern of errors shows that fast vehicle travel may upset these estimations of distance traveled on a particular leg or angle, and indeed some of the most accurate measurements that I have come from within a cave mouth in dense bush, a location nevertheless reached by foot.

\section{Memory}

The hypothesis is that GY speakers code scenes for memory complete with directional information. The reasoning behind the hypothesis is this:

1. GY speakers use, indeed (in the absence of other descriptive resources in the language) must use, absolute directions to describe spatial locations and events;

2. in order to be able to do so, they must accurately remember scenes together with their cardinal orientation.

A loophole in the reasoning, though, is that it is possible that GY speakers simply make up the necessary directions, when these are not clear from familiar or perceptual surroundings. Just as English requires me to specify singularity or plurality, so that I might say, "I saw a man lurking in the alley," even when I am unsure whether there was one or two, perhaps GY speakers simply impose an orientation on descriptions when the language requires it.

It is in fact fairly straightforward to demonstrate the accuracy of GY spatial descriptions from memory. For example (following similar exercises by Haviland), I have examined filmed stories about events that happened 
long ago, involving complex journeys and movements over terrain. The sequence of events may be related precisely to a map, their internal coherence checked, and the descriptions of locations verified against reality and other peoples' memories. The precision of gestural indications of angle may also be checked up to the limits of estimation from a film record. In all cases, the linguistic and spatial specifications seem to check out with remarkable reliability. The interpretation of both directional terms and gestures is not though a simple matter, as they are often (but not always) from the point of view of the protagonists at that particular point in the story (Haviland 1996).

An especially convincing case study by Haviland (1993) serves to make the point best. Haviland filmed a man in 1980 telling a story about how he and a companion were shipwrecked in a ketch. In 1982 I serendipitously filmed the man retelling the same story. By comparing the two tellings, event by event, Haviland shows that orientations are preserved across the two tellings down to the details of which way the ketch rolled over, on which side the two men jumped out, who was west of whom on the swim back to shore, and so on, throughout the story.

For this study, further attempts were made to explore the nature of memorized directions. On one occasion, a group of about a dozen Hopevale men made an overland trip to Cairns, the nearest city, 250 kilometers to the south, to discuss land rights issues with other Aboriginal groups. The meeting took place in a room without windows, in a building reached either by back alley or through a car park, so that the relation between building and city layout was somewhat obscured. Between a month and six weeks later, I asked half a dozen of them (as I happened to meet up with them) about the orientations of the rooms and speakers at the meeting, and also details of their hotel rooms. They could all give immediate, and as far as I was later able to ascertain, accurate responses to these questions. ${ }^{23}$ In particular, there was complete agreement about, for example, the orientation of the main speaker, the blackboard, the breakfast room at the hotel, and so on.

Somewhat more systematically, ten informants were shown a six-minute film, depicting various events without words, and asked to describe it to another native speaker. ${ }^{24}$ Although there was no systematic delay between watching the film and describing it, the length of the film made it likely that at least a six-minute interval arose between watching and describing the relevant portion of the film. Every one of the descriptions obtained includes many orientational specifications, which are correct for the virtual space behind the screen. (If a man appears to be coming toward the viewer and the viewer faces south, then the man will be described as coming northward.) Or as one young man remarked on another occasion, "You always know which way the old people been watching the TV when they tell the story."

Memory for visual percepts should be an especially interesting issue to investigate. Clearly we cannot see cardinal directions, and our visual system must process information in terms of relative position, for example, locating objects in the left or right, or foreground or background, of our visual field. So we expect visual perception to operate in terms of a relative, viewpointcentered system. But equally clearly we cannot see without looking in a 
particular direction, and it is conceivable that visual percepts are coded for memory by GY speakers with that direction attached.

As a first attempt to investigate this, 12 Polaroid snapshots were taken of chairs on the local school playing field. Three shots were taken in each of four directions, clearly recoverable by the mountains or other features in the backgrounds. For each background, two chairs of identical shape were arranged side by side, in three different arrangements: (a) white chair on the left, red on the right, (b) red chair on the left, white on the right, (c) two red chairs side by side. The snapshots were shuffled like a pack of cards, and the informant asked to pick one and remember it carefully. Then it was put back in the pack, reshuffled, and the informant was asked to find it by looking through the pack. While the informant was doing so, verbal protocols were obtained, in order to find what principles were being applied. The informants mostly spoke in English. The protocols revealed that, although some informants searched for a named background (e.g., Nugal Mountain) and then, say, for a white chair on the left, most did indeed seem to have used the background as a clue to the cardinal direction of the shot and, so, could think about the white chair as, say, to the north. The experiment was not pursued because it relied on a verbal protocol, and for an investigation of the relation between language and cognition it seemed better to develop a direct test of whether memory was by cardinal direction or relative notions like left and right, whether verbalized or not. It is to these investigations we now turn.

Recognition Memory. Visual perception and processing is universally viewpoint dependent, or relative in nature. But a visual percept may be coded for long-term memory together with viewpoint-independent (cardinal direction) information by GY speakers. This would transform the percept into a fundamentally different concept, with testable consequences. For example, if a subject facing north is presented with a card on which there is red square to the left (and thus to the west) and a blue rectangle to the right (and thus to the east) and then the subject is turned around facing south and presented with two cards with a mirror image arrangement of red square and blue rectangle, which card will he or she identify with the first stimulus card? If the subject chooses the card with red square to the left, the red square will now be on the east side, but if the subject thought about the original stimulus as red to west, he or she will choose the westward red square even though it now is on the right (see Figure 1).

Following this line of reasoning, a series of tests was developed. They were conducted in a pair of rooms that were interconnected but so arranged that they were mirror images of one another, down to the last detail of the furniture. ${ }^{25}$ So from the interconnecting door, each room visually presented the identical scene, with the desk at the right hand on the far wall. One desk thus faced north, the other south (see Figure 2). The passage between one desk and the other, around the existing furniture, took approximately $30-45$ seconds, so that together with verbal instructions about one minute passed between a subject seeing a stimulus set on one table and the identical set on the other table. This setting gave the minimum environmental encourage- 


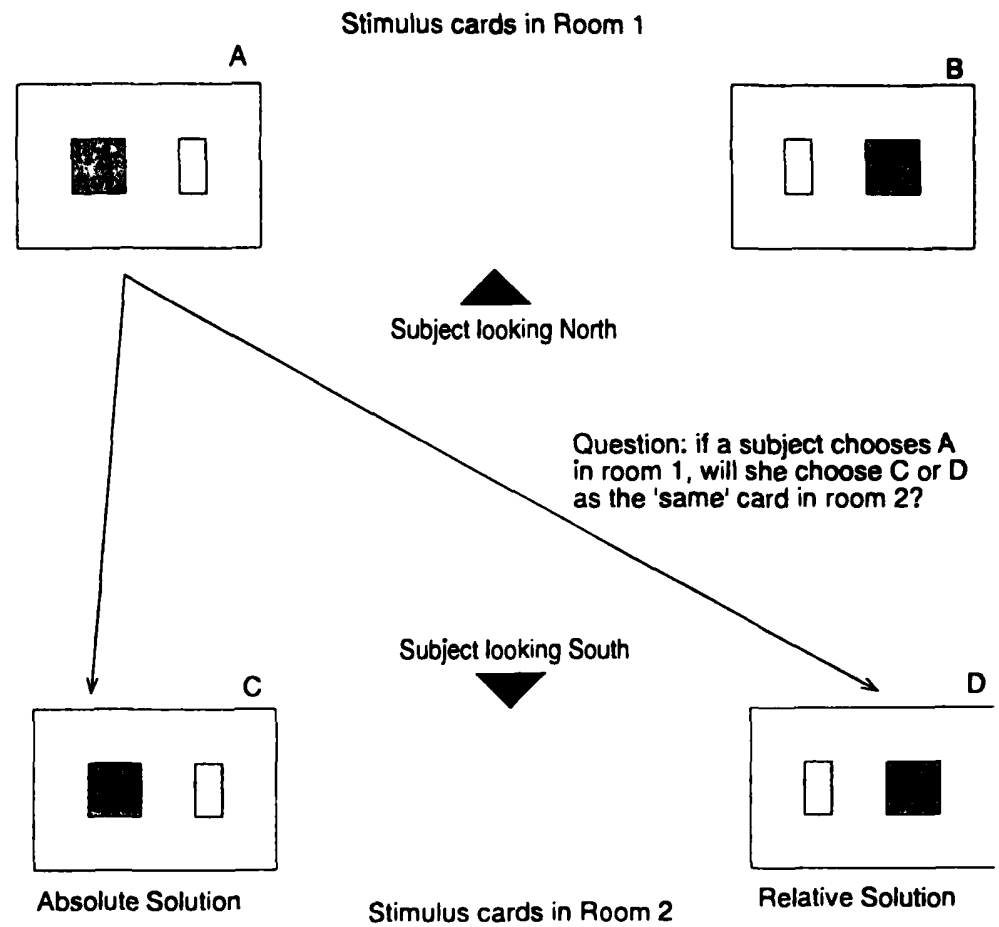

Figure 1

ment to thinking in terms of cardinal directions, compared for example to carrying out experiments in the bush or on the beach, and the mirror-image rooms gave a definite bias toward thinking in relative terms, for example, in terms of left and right. Thinking in terms such as "toward the bed" or "toward the cupboard" in such mirror-image settings would also yield the same results as thinking in terms of left and right. Again in order to not prejudice the results, all instructions were given in English, and the subjects

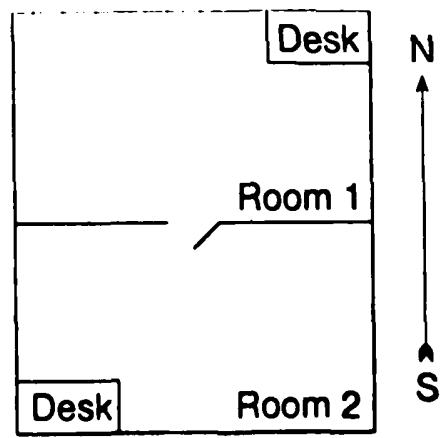

Figure 2 
therefore responded in English where a verbal response was requested. This minimized any possible residual effect of "thinking for speaking" (Slobin 1996), even though the main task set to informants was nonverbal: they had merely to indicate what they previously saw by pointing.

The simplest of the stimulus sets consisted of a pair of two (i.e., four) black cards, each of which had glued on it two chips of plastic: one bright red, about 2 centimeters square, one light blue about 1 centimeter wide and 2 centimeters long. The size difference of the chips was designed to guard against any unknown effects from color blindness. The chips were identically arranged on each card with exactly the same spacing between them and the edges of the card, using a template. Each pair of cards was displayed as follows. One card was placed above the other but twisted around, so that the top card had the red chip to the left and the blue to the right, while the bottom card had blue to the left and red to the right. The initial pair, or stimulus set, was displayed in room 1 on the desk viewed looking north, while an identical arrangement (top card with red to the left) was placed in room 2 on the desk viewed looking south.

Subjects were led into room 1 and asked to choose one of the two cards. Having chosen it, they were asked to remember it. Then they were turned around and led to room 2 , where they now faced the identical arrangement facing in the other cardinal direction. They were then asked to identify the card that they chose before. They were then told to keep remembering it and were led back to room 1 where they saw the previous stimulus set rearranged, so that the card that was on the top was now on the bottom. Again they were asked to identify the previously chosen card. Finally they were led back to Room 2, where they were shown the same two cards, now placed side by side rather than on top of each other (see Figure 3 ). The three trials were intended to test for consistent responses. In cases where consistent answers were obtained, verbal protocols were sometimes also collected afterward, in response to the question "Well done, but how did you do it?"

The hypothesis was as follows: Subjects might identify the cards that had, for example, the red chip to the west, rather than two cards with chips each to the left so that one (viewed looking north) had the red chip to the west and the other (viewed looking south) had the red chip to the east.

The first rows of Table 3 and 4 display the results. (The second rows describe results from the same experiment run on a Dutch sample, to be described below.) A transition between rooms 1 and 2, or 2 and 1, which maintained the left/right relation of the chips was coded as relative (identification by a relative system), a transition that maintained the absolute direction of the chips was coded as absolute. There were 12 subjects, of whom ten completed the three trials. All were men aged 40 or over. Of these, seven subjects made consistent choices over the three trials based on the east/west location of the chips, that is, on absolute orientation rather than relative left/right location (see Table 4). Only one subject showed a consistent left/right strategy, but he only did two trials. A sheer count of absolute versus relative identifications yields a ratio of 27 absolute to 7 relative, or an overwhelming imbalance in favor of an absolute strategy for remembering the disposition of the chips. 


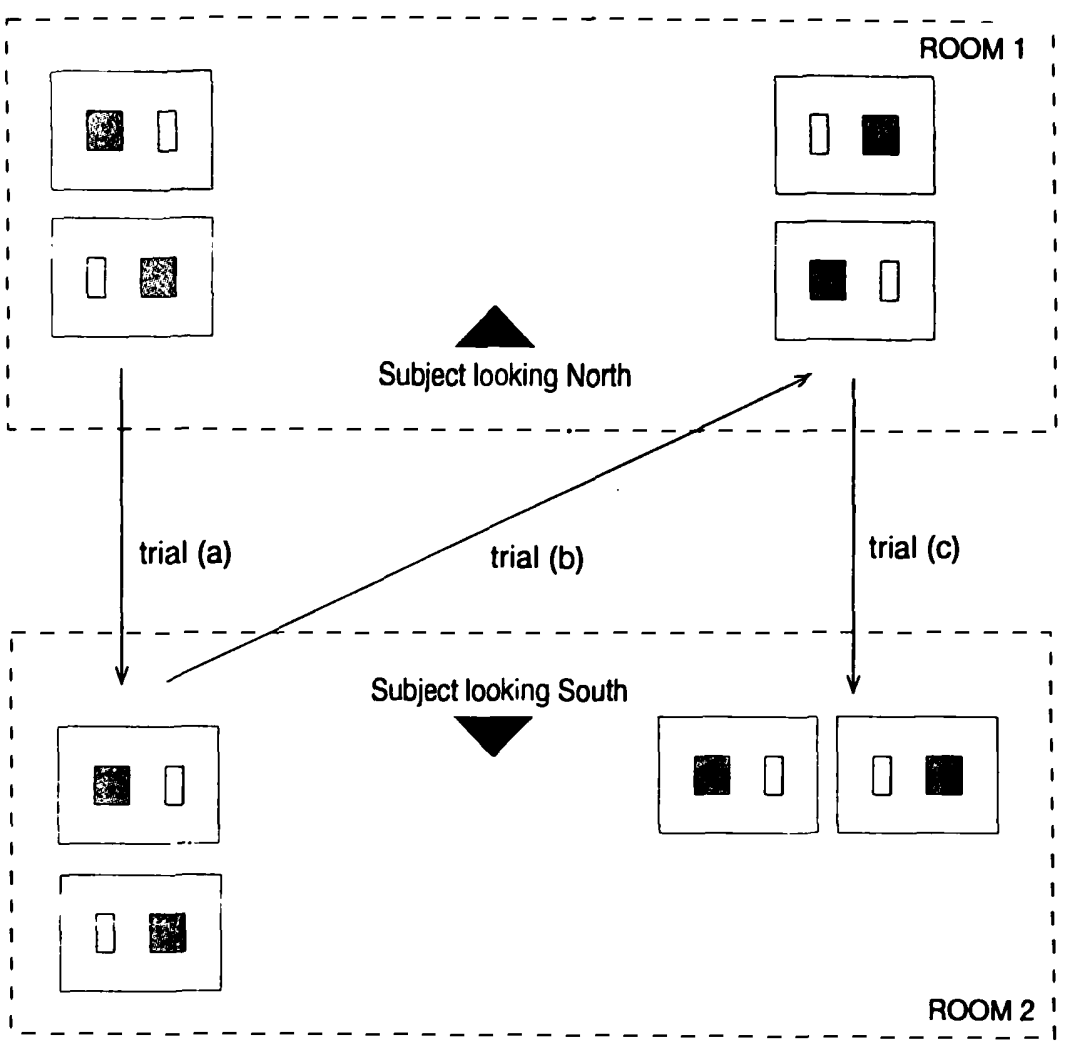

Figure 3

The results clearly show that the majority of Hopevale subjects identified the chosen card on grounds of absolute orientation; they identified consistently cards with say blue chips to the east. Verbal protocols volunteered by some of them confirmed that this was the underlying criterion for identity. Only one subject may have consistently chosen cards on the basis of, for example, red chip to the right-hand side. Four subjects either made

Table 3

Orientation by means of which individuals identified memorized chips.

\begin{tabular}{lccc}
\hline \hline & Absolute & Relative & Total \\
\hline Hopevale & 27 & 7 & 34 \\
Dutch & 1 & 44 & 45 \\
\hline
\end{tabular}

Hopevale versus chance: $p=0.0004$ (binomial test, $P=0.5$ for absolute, relative); Dutch versus chance: $p=0.0000$ (binomial test, same assumptions); Hopevale versus Dutch: $p=0.0000$ (Fischer's exact test). 
Table 4

Consistency of subjects memorizing chips, over the trials

\begin{tabular}{lcccc}
\hline \hline & Absolute & Relative & Inconsistent & Total \\
Hopevale & 7 & 1 & 4 & 12 \\
Dutch & 0 & 14 & 1 & 15 \\
\hline
\end{tabular}

errors between the three trials or switched systems from an absolute to a relative one midstream.

For comparison, the same experiment was run on 15 Dutch subjects (of both sexes and mixed ages). The results (see the second row of Table 3 ) show an overwhelming preference for identification on the basis of left and right (thinking about the red chip as, say, to the left). The binomial test of independence shows that the Hopevale subjects have a significant preference for absolute choices $(p<.001)$, while the Dutch subjects have a significant preference for relative choices $(p<.001)$. Fischer's exact test of independence confirms that the two subject pools taken together differ significantly with respect to their orientation preference $(p<0.001)$.

One may also compare individual behavior across the two groups, by contrasting the number of individuals who had a majority of choices absolute or relative (see Table 5). Since two of the Hopevale subjects only made two choices, we leave them aside and compare those individuals who had two or more of three choices in one category or the other. Again we find, using the same techniques as before, that the preferences of Hopevale subjects for absolute choices are significant $(p<.02)$, as are the preferences of Dutch subjects for relative choices $(p<.001)$. The difference between the two groups in preferred orientation is also significant $(p<.001)$.

To conclude this section, these experiments (together with another reported in Levinson n.d.[a]) do suggest that GY speakers code visual arrangements for memory in absolute terms, not purely in terms of the viewpoint perspective given by visual perception. The mixed results show that recognition with small time delays may also tap into a viewpoint memory, a mental "snapshot" as it were, but that is surely not surprising. It would be interesting to repeat these tests with much longer time delays.

Table 5

Majority orientation by means of which individuals identified memorized chips.

\begin{tabular}{lccc}
\hline \hline & Absolute & Relative & Total \\
\hline Hopevale & 9 & 1 & 10 \\
Dutch & 0 & 15 & 15 \\
\hline
\end{tabular}

Hopevale versus chance: $p=0.0107$ (binomial test, $P=0.5$ for absolute, relative); Dutch versus chance: $p=0.0000$ (binomial test, same assumptions); Hopevale versus Dutch: $p=0.0000$ (Fischer's exact test). 
The results do suggest though that GY speakers perform nonverbal recognition tasks in potentially different ways than those who speak languages that make predominant use of relative spatial coding.

Recall Memory. We have described some nonverbal recognition tasks. We now turn to consider nonverbal tasks that require more than recognition of a prior stimulus. We have already described some verbal recall tasks, for example, where informants were asked to describe the content of a film or to report the orientations of the meeting at Cairns. Here we report on a simple experiment. Subjects were shown a lineup of a toy man, a pig, and a cow on the desk in room 1, with a northerly viewing angle. The line headed east, or toward the subject's right. The subject was told to memorize the assemblage, so that if the experimenter put the figures in a heap, the subject could reconstruct it. He was then led into room 2, facing the desk looking south, presented with three identical figures in a heap and asked to arrange them just as before.

Most informants interpreted the problem in such a way that they concentrated on the relative order of man, pig, and cow and their relative distances. Some informants arranged the line facing east; that is, they remembered the arrangement in terms of absolute direction in which case the response was coded absolute. Some arranged the line facing west; that is, they preserved its relative direction toward the subject's right in which case the response was coded relative (see Figure 4). The results are shown in the first row of Table 6.

Nine out of 15 informants chose to preserve absolute orientation and jettison relative (rightward) orientation of the lineup. All the remainder chose to preserve relative orientation, that is, the rightward direction of the lineup. Note that if there were physically only two alternatives here, the result would be scarcely better than chance. But although subjects might have arranged the lineup in any cardinal direction or any relative direction (e.g., away from themselves), only a northward or rightward solution was adopted.

Given the recognition experiments and the predominance of absolute results there, I had expected more decisive absolute results here, whereas in fact six informants took a relative perspective. Nevertheless, those taking an absolute perspective are unlikely to be doing so by error, as shown on some occasions by volunteered verbal protocol. In a pilot precursor, the arrangement was more complex with a tree behind the line of animals in

Table 6

Alignment strategy for placing animals in a row.

\begin{tabular}{lccc}
\hline \hline & Absolute & Relative & Total \\
\hline Hopevale & 9 & 6 & 15 \\
Dutch & 2 & 13 & 15 \\
\hline
\end{tabular}

Hopevale versus chance: $p=0.3036$ (i.e., nonsignificant) (binomial test, $P=0.5$ for absolute, relative); Dutch versus chance: $p=0.0037$ (binomial test, same assumptions); Hopevale versus Dutch: $p=0.0105$ (Fischer's exact test). 

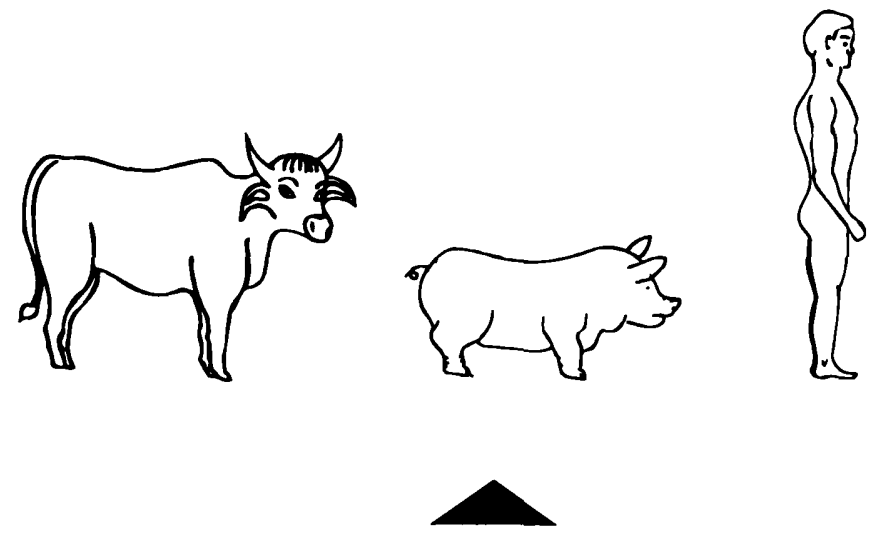

Subject, facing North

Room1

Room 2

Subject, facing South
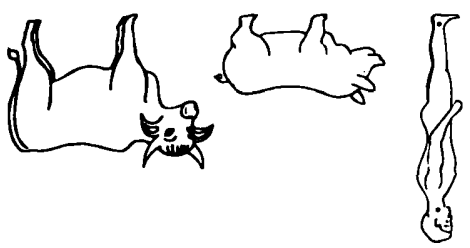

Absolute Solution
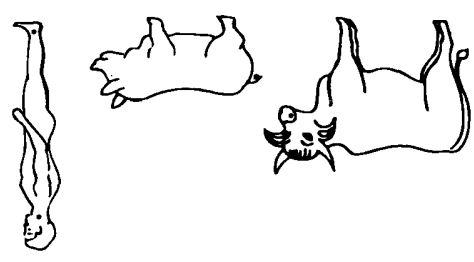

Relative Solution

Figure 4

room 1 . Two out of three subjects not only arranged the line absolutely but also put the tree in front of the line in room 2, clearly demonstrating a retention of absolute orientation. For comparison, the same experiment was run on Dutch subjects (see the second row of Table 6).

The binomial test shows that the Dutch subjects have a preference for relative arrangements ( $p<.005)$. But for the Hopevale subjects there is no statistically significant preference for absolute (eastward) arrangements 
over relative (rightward) arrangements of the figurines. A larger sample of course might have confirmed the slight trend toward absolute. But since there were no other arrangements, the distribution at least confirms that Hopevale subjects chose between these two solutions. The Fischer exact test confirms that the difference between the two subject pools is significant $(p<.02)$.

\section{Inference}

Might it be possible to show that, not only in memory tasks involving recognition or recall but also in various higher but nonverbal cognitive processes, essential recourse is had to absolute orientation by GY speakers? In a pilot task, informants were presented with a set of about 20 cards. Each card had a photograph of a familiar building or other site on the Mission, for example, the store, church, cemetery, sawmill, and so on. On the back of the card was a photo of the same building from the other side. The cards were supplied with stands so that they could be set upright. Four informants were given the cards and asked to arrange them like a map of the mission site without further instruction. In all cases, not only did the informants arrange the cards in the correct absolute orientation, so that, for example, the cemetery was south (and slightly east) of the hospital, but also the informants oriented the individual cards so that the front of the church faced west, the back of the hospital east, the back of the store south, and so forth, thus matching reality.

This seems to show that, whereas for us a map or plan has an arbitrary orientation, for GY speakers any such plan should be correctly oriented. This was also demonstrated on various occasions by sketch maps drawn in the sand. For example, on one occasion when talking over the challenge mounted by one highly educated Hopevale resident in the state high court to a land rights decision on the Quinkan reserve, he drew the map of the reserve lands in the dust on the table in a workshop. I was confused until I realized that he had located south at the top of the map, because that was also the true direction.

Thinking like this might lead to distinct solutions to inferential problems, along the lines of Johnson-Laird's (1983) theory of mental models. To test this, four mazes were drawn on large cartridge sheets. Each maze had a black circle as starting point from which began a path made of equal length arrows (each $10 \mathrm{~cm}$ ), each following the other either in a straight line or at $90^{\circ}$ angles. Thus a rectilinear path was traced, like the path through and round half a dozen city blocks (see Figure 5 ).

The path came to an end at (variously) two or three arrow lengths from the starting circle. The task was to choose from a set of three cards the one that would complete the path back to the starting point. Two of the cards were identical, except that one was rotated $180^{\circ}$; both of these cards were acceptable solutions to the puzzle. The third card was a blind or distractor; there was no way that this one could lead back from the end of the path to the starting point. The idea was to show the maze in one room, facing in one direction, and then to take the subject into the other experimental room facing the other direction and ask him to choose the correct card. The 
question was whether he would preserve the absolute orientation of the maze and thus choose out of the two correct cards the one that retained the absolute direction, or whether he would choose the card that was the correct solution from a relative visual perspective. In either case, the problem was

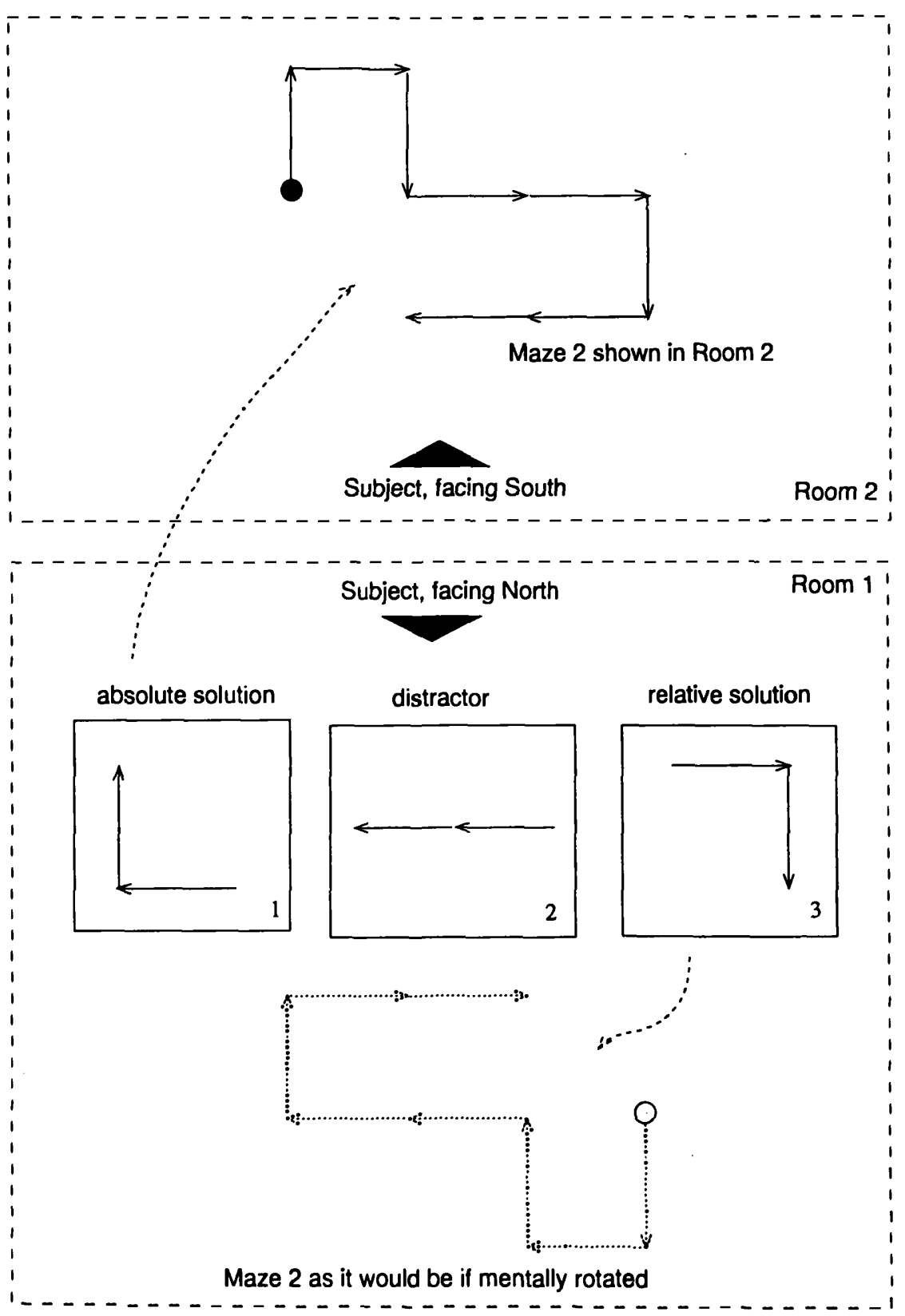


Table 7

Orientation by means of which individuals completed mazes.

\begin{tabular}{lcccc}
\hline \hline & Absolute & Relative & Blind & Total \\
Hopevale & 24 & 11 & 1 & 36 \\
Dutch & 3 & 42 & 0 & 45 \\
\hline
\end{tabular}

Hopevale versus chance: $p=0.0000$ (multinomial test, assuming $P=$ 0.3333 for absolute, relative, blind); Dutch versus chance: $p=0.0000$ (multinomial test, same assumptions); Hopevale versus Dutch: $p=0.0000$ (chi-square).

not simply visual, since there were always at least two or more possible paths, only one of which was represented by the two correct cards. The subject had to mentally compute a set of possible answers and then see if the cards matched. It thus contained an essential inferential element, and indeed it is not an easy task regardless of how it is achieved.

Subjects were shown the mazes in room 2, facing south. The task was demonstrated on one maze that was not further used in the experiment (Figure 5). The cards of the demonstration maze were shown in the same room, and it was shown that (holding their orientation constant) only one of them would complete the maze. When the subject had the hang of it, the second maze was displayed, and the informant told to memorize it. He was then led to room 1 and shown the three cards on the desk that he faced looking north. If he had conceived of the maze as oriented absolutely, he should pick the card whose orientation if maintained exactly would complete the path. If he conceived of the maze as a pathway of relative turns and straights, he should choose the card that would complete the maze if he mentally had rotated the maze along with himself. The subject was asked to take his time and chose the card that would complete the path. He was permitted to return and have another look if he wished (mostly this was not taken up). On the whole, subjects decisively and immediately chose a card. Each subject performed three such maze tasks, to see if a consistent pattern arose for each subject. (See Figure 6 for diagrams of the mazes.) The results are listed in the first rows of Tables 7 and 8 .

Only one choice of an impossible path (the blind) was made, suggesting that the task was properly comprehended. Out of 12 subjects there were 5 wholly consistent absolute-strategy users and only 1 consistent relative (left/right) strategy user. As shown in Table 9, 8 out of the 12 subjects had a majority of absolute choices. The ratio between trials displaying an

Table 8

Consistency of individuals over three trials of maze completion.

\begin{tabular}{lcccc}
\hline & Absolute & Relative & Inconsistent & Total \\
Hopevale & 5 & 1 & 6 & 12 \\
Dutch & 0 & 12 & 3 & 15 \\
\hline
\end{tabular}


absolute strategy, a relative strategy, and choosing an erroneous solution was 24:11:1. The results show that the Hopevale subjects were performing on a nonrandom basis over the three trials and that the majority of consistent users were absolute thinkers in the ratio of 5 absolute to 1 relative subject (even if the test in Table 9 of Hopevale subjects by majority of choices failed to reach statistical significance).

The same experiment was conducted on 15 Dutch people (of various ages and both sexes). The results are overwhelmingly in the direction of relative
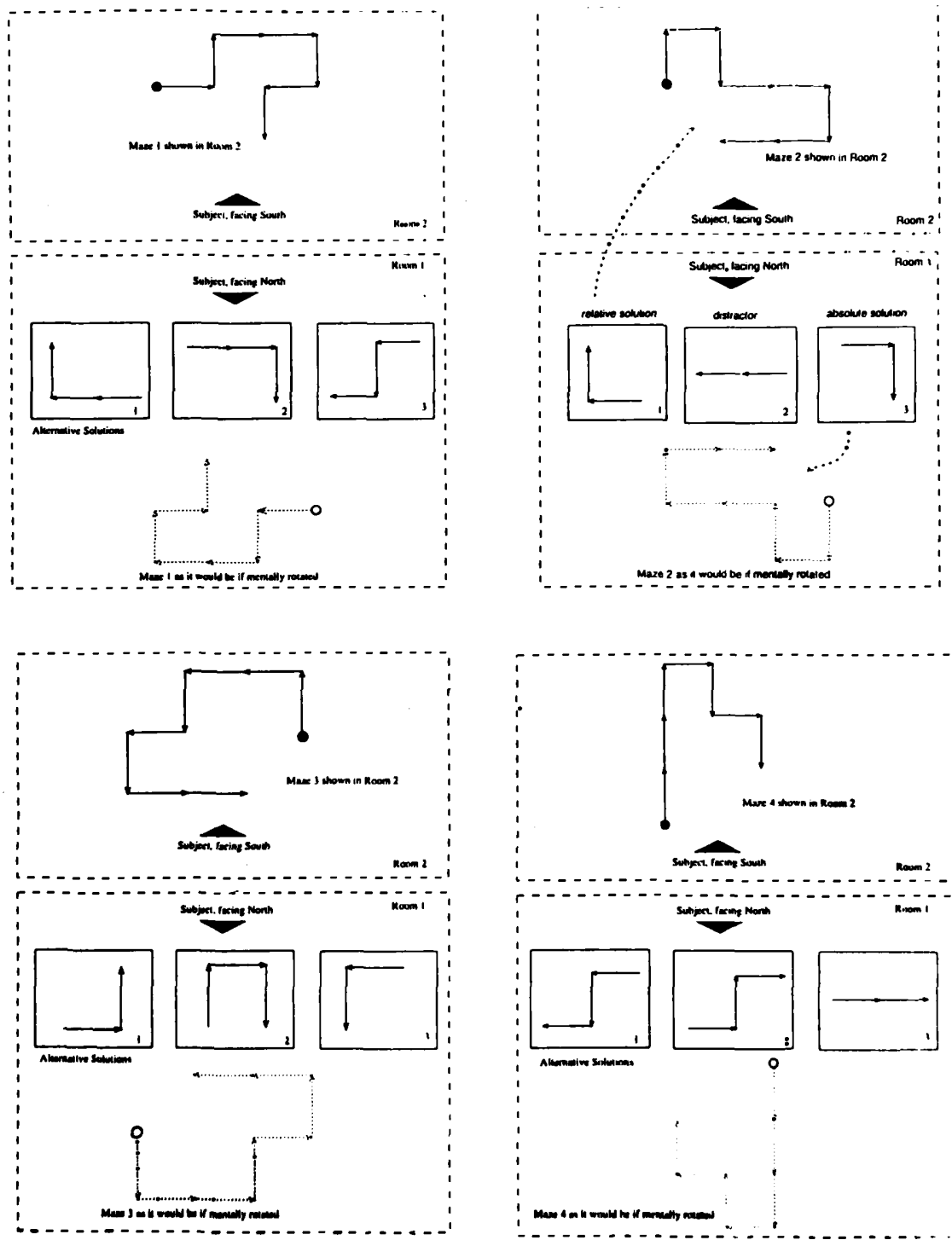


\section{Table 9}

Majority orientation by means of which individuals completed mazes.

\begin{tabular}{lccc}
\hline & Absolute & Relative & Total \\
\hline Hopevale & 8 & 4 & 12 \\
Dutch & 0 & 15 & 15 \\
\hline
\end{tabular}

Hopevale versus chance: $\mathrm{p}=0.1938$ (binomial test, $\mathrm{P}=0.5$ for absolute, relative); Dutch versus chance: $p=0.0000$ (binomial test, same assumptions); Hopevale versus Dutch: $\mathrm{p}=0.0002$ (Fischer's exact test).

thinking (see the second rows of Table 7, 8 and 9). The multinominal test of independence shows that there is a significant preference for absolute choices by Hopevale subjects $(p<.001)$ and for relative choices by Dutch subjects $(\mathrm{p}<.001)$. A chi-square test shows that the preferences of the two subject pools differ significantly $(p<.001)$ with respect to absolute, relative, and blind alternatives. ${ }^{26}$

\section{The Experiments: Discussion}

As with all the parallel Dutch experiments, the maze result by Dutch subjects is more decisively "relative" than the Hopevale results are decisively "absolute." There are a number of possible reasons for this. One would be that the more educated, younger Dutch sample (which included university students) found the tasks less bizarre; they may also simply have better memory ability than the Hopevale sample of aging men. The experimental conditions were also not completely matched, in that the rooms were of a smaller scale in the Dutch case, so that effectively less time and less travel intervened between seeing the first stimulus and manipulating or seeing the second. But it is unlikely that these factors account for the variance.

One line of explanation might focus on the fact that GY speakers are bilingual; they must therefore remember scenes for possible description in both GY and English. Since idiomatic description in English will be in terms of left/right, front/back, this will require coding for memory in relative terms. Thus GY speakers will have available both kinds of codings in memory. Since the experiment instructions were in English (to avoid predisposing subjects to "thinking in GY"), with about a third of informants this may have triggered use of the relative coding in a nonverbal task. There are some problems with this explanation, one of which is that Hopevale English makes very little use of relative spatial descriptors such as "to the left of." Another is that it does not explain differences across tasks. A third is that memory encoding in absolute terms might alone be sufficient, since one can generate viewpoint-dependent descriptions from viewpoint-independent ones, but not vice versa (see Levinson 1996a). Bilingualism may nevertheless be one part of the explanation.

Another part of the explanation, I suspect, is the following. In the Dutch or, indeed, English case, the kind of coding provided by our visual system 
(e.g., left in the visual field) coincides with, or at least is similar to, the kind of coding required by linguistic production (e.g., "the red chip is to the left"). The latter requirement constrains how we remember things, to allow for later possible speech encoding. So how we English speakers code scenes for long-term memory is consistent with how we code them visually and linguistically. In the Guugu Yimithirr case, visual encoding will be relative, or viewpoint-dependent, as with us, but linguistic encoding is viewpointindependent or absolute ("the red chip is to the west"). It is likely that, at least for short-term memory, both kinds of coding are available and accessible since, for example, motor control is surely guided by the viewpointdependent information. Long-term memory may be exclusively absolute in nature (allowing computations of viewpoint-dependent angles) or more likely mixed (especially to the extent that it is visual). In other words, when a Hopevale man wakes in the night and searches for the alarm clock, it is an open question whether he thinks, "It must be just there by my left arm," "... just north there," or both. What we do know, on the basis of the experiment and anecdote here reported, is that the absolute coding is almost certainly available to the GY speaker, and it is almost certainly not readily available to the Dutch speaker.

The mixed nature of the GY results reported above might therefore have the following explanation. GY speakers have available both kinds of memory encoding for visual scenes: viewpoint dependent and absolute. The absolute coding may be especially prominent for long-term memory. Our tasks, with relatively short memory intervals (one minute plus or minus 15 seconds), tapped into both. Other tasks not reported here (see Levinson n.d.[a]) suggest dual coding; thus a task with longer memory interval showed more decisive use of an absolute system of coding, while a task involving recognition discrimination between mirror-image line figures seems to have tapped more in to the relative system. Dutch speakers generally seem to have no such duality of systems at their disposal, and the occasional choice of an absolute response may be due only to error.

The idea that the visual coding system and the linguistic coding system, and their associated memory requirements, might be incongruent in some cultures and not in others is important. It suggests for one thing that there might be substantial differences in child development cross-culturally. Alternatively, it raises the following speculation: Piaget notes the very long developmental gap between perceptual abilities (e.g., of concepts of "front," "behind," "left," and "right") and the corresponding conceptual abilities. But what accounts for this strangely laborious recapitulation on the conceptual level of what is so effortlessly given on the perceptual level? One solution to the puzzle may be that the Indo-European languages just happen to display an isomorphism in spatial perception and conception, which is absent from many languages, including GY. Thus children cannot be so structured as organisms to presume such an isomorphism but must slowly find out whether things are so (if their language is English) or not so (if their language is GY). 


\section{Directionality and Imagination}

If among GY speakers scenes are normally remembered with orientational information and inferential processes are adapted accordingly, the intriguing possibility arises that even hypothetical or imaginary scenarios are thought of as oriented in particular ways. ${ }^{27}$ It is hard to know how to investigate this seriously, but a series of pilot probes were tried as follows.

First, informants were asked to translate verbally three passages, two from the New Testament and one adapted from a newspaper story about a car chase. The New Testament passages recounted Jesus' trip across the Sea of Galilee and his casting out of the demons of Mob (Mark 4:35-5:11) and the miracle of the good catch (John 21). Three exemplars were obtained, and each imposed an arbitrary orientation upon the lake crossing and subsequent events and upon the fishing expedition. Thus for example the passage "Let us go to the other side of the lake" was rendered (in gloss) "Go to the east side of the sea," and the passage "Throw your net out on the right side of the boat" was rendered (in gloss) "Throw your net out on the east side of the boat." The car chase with left and right turns was more problematic, but again recourse was had to an arbitrary imposed orientation.

It may be complained that this imposition of cardinal directions on a scene shows nothing about conception, only about the necessity of making additional assumptions for adequate translation (just as, when translating you into French, I must choose between $t u$ and vous). So another kind of task was assigned, namely to provide generalized recipes for actions of various kinds. For example, five informants were asked to describe how to catch the large seagoing turtles that are prized as festive eating by Hopevale people. The procedure involves chase by a dinghy until the turtle surfaces for air, whereupon it is either harpooned or grabbed by a man diving overboard and wrestled to the surface and into the boat. Again all the verbal recipes or instructions were given to me in GY in an oriented way, so that the turtle was visualized, or at least described, as being on a particular side (east, etc.) of the imaginary boat that we were sitting in.

Perhaps under such circumstances, informants naturally think of some particular actual event in which they participated, thus accounting for the concrete instantiation of the generalized instruction. Therefore inquiries were also made about dreams. Four dreams were collected, either wholly or partly in GY. Each had some motion description or involved some imaginary landscape (e.g., heaven), and each account oriented the motion or landscape in specified cardinal directions. I am glad to be able to report that heaven is entered heading north (at least from Cape York) and that you will be met by the Lord (half Aboriginal) coming south toward you!

Although none of these probes are decisive, they are at least consistent with the possibility that GY speakers supply absolute orientations to historical, hypothetical, or entirely imaginary scenarios. ${ }^{28}$ If their recognition, recall, and especially inferential faculties habitually make use of absolute orientational information, then it would make sense to think of hypothetical scenarios in the same, directional way. 


\section{Conclusions}

There are many caveats that must be made about the experiments reported here. They were, for reasons mentioned in the introduction, conducted on a very small sample of informants, namely, those relatively senior men whose sociolinguistic prowess in Guugu Yimithirr was undisputed and who happened to be available in the community and willing to help at the time of study. Because the experiments were conducted over a number of weeks and because linguistic work was proceeding concurrently with a few of the same informants, the interests of the experimenter (though bizarre) must have been discernible to some (but not all) subjects. In addition some further pilot experiments were begun but never completed, giving further exposure to the small sample of subjects. On the other hand, considerable noise was introduced into the results by interruptions, and lack of interest or other distractions. My judgment is that the results do tap into genuine patterns and that a deeper study on a wider sample would confirm the results reported here.

Despite these limitations, if these results are taken together with the many qualitative studies (especially by Haviland) that we have for this community and with the kind of anecdotal evidence partially reported here, they do point in a single convincing direction. Not only do Guugu Yimithirr speakers, at least of this older generation, speak a language that as a prerequisite requires storage and computation of orientation and absolute directions, but they can also be shown when not engaged in speaking the language to think in a way that is concordant with it. This represents a serious challenge to the view that a particular language at most requires a special way of thinking just while speaking. Rather, the fact that GY provides an absolute system and no system of relative description seems to have further effects: speakers must remember spatial dispositions in the absolute terms that will allow them to later code them in the language. Spatial memory will then determine an absolute mode of spatial inference. Absolute coding in both memory and language in turn requires a constant, background "dead-reckoning" of current heading and position, which our pointing experiments appear to have tapped directly into. Language, gesture, cognitive style, and many aspects of spatial behavior, come to form a coherent and distinctive complex.

It may be objected that the whole system of absolute orientation, the associated gesture and interaction system, is much more than merely a linguistic phenomenon and therefore cannot be considered an example of linguistic determinism. Why not, for example, reverse the argument and claim that the cognitive system of absolute spatial conception drives the language? The answer is that there is no obvious way in which a community-wide cognitive practice of this sort could come to be shared except through its encoding in language and other communicative systems like gesture (see Haviland 1993). It is the need to conform to these communicative systems that requires convergence in cognitive systems, not the other way around.9 
Finally, what do Hopevale residents think about this peculiar ability of theirs? They are aware of the inability of most wangarr ('spirits' or white people) to think this way, and when speaking English very rarely use the English cardinal direction terms (although they gesture freely in a way that presupposes absolute orientation). Conversely, it is locally well known (my colleague Lourdes de León reports) that children in school have enormous difficulties with left and right. But there does not seem to be any general realization that this system constitutes an intellectual achievement of the first order and one of the central surviving features of a culture under prolonged erosion, which connects to that enduring traditional preoccupation of all Aboriginal peoples, the landscape, and its hidden meanings.

\section{Notes}

Acknowledgments. This article reports on fieldwork conducted from July to September 1992 in Hopevale Aboriginal community, Cape York Peninsula, in northern Queensland, Australia. I had the benefit of much help from John Haviland and Lourdes de León, who shared the first three weeks' fieldwork and gave me many useful ideas about how to pursue the inquiries reported here. I am grateful for the tolerance, interest, and support from the community at large and, especially, from many helpers and consultants, too numerous to name. But I am especially grateful to Roger Hart, who helped me transcribe large amounts of Guugu Yimithirr text with exemplary patience and to the residents of the Hopevale Rest Home, who put up with many pilot experiments. Dan Charlie, a constant companion on field trips, made sure I never got lost in the bush, which given my natural ineptitude was quite a feat. Walter Jack was instructive company on many trips, and Jimmy Hart provided essential crab and refuge at the beach. John Haviland's $(1979,1993)$ earlier grammatical work on the language made all of this possible.

I later received much help from colleagues, and my thanks are due to Harald Baayen for handcrafted statistical programs, to Gertie de Groen for further help with the diagrams and statistics, and to Edith Sjoerdsma for editorial assistance. I received useful comments on versions of the article from Penelope Brown, Eve Danziger, John Haviland (twice), Eric Pederson, Dan Slobin, Pieter Seuren, and the editor and referees of this journal, but remaining errors are of course my own. In general, the direction of research reported here owes a great deal to the provocative ideas of John Lucy (1992), and Dan Slobin (1991, 1996).

A somewhat longer version of this article was circulated by the Cognitive Anthropology Research Group in 1992 (Levinson n.d.[a]). I have not undertaken a more substantial revision for a number of reasons. First, the earlier version, now unavailable, has been much referenced and was the origin of many later ideas and methods. Second, although we have now developed more sophisticated experimental methods (see, e.g., Levinson 1996a), there is little chance of my returning to Hopevale to employ them. Third, the linguistic background to this study, only sketched here, is fully developed in Haviland's article in this journal (1993), which demonstrates how complementary findings may be obtained from the careful observation of gesture. His article, thus, compensates for gaps and failures in my own, and should be read as an essential companion piece.

1. For a cross-linguistic typology and more careful exposition of the "relative" / "absolute" contrast, see Levinson 1996a.

2. Guugu Yimithirr has terms for left hand and right hand, but these are only body-part terms. Other body-part terms, such as "nose" may be used in expressions 
glossing as "nose to north," and so forth, to indicate orientation of a body, but they play no other essential role in spatial description. The terms for "up" and "down" share some of the morphological properties of the cardinal directions and may be thought of as belonging to the absolute system. (They are in fact used locally as cardinal directions; see Haviland 1979:76.) There are terms for "inside," "outside," "underneath," "in between," and so forth, together with locative, ablative, and allative cases. But there is nothing remotely similar to our familiar left/right/front/back system (see Haviland 1993).

3. I have rehearsed these arguments in more detail in another article based on another unrelated language, which also makes use of an absolute system (Levinson 1996a).

4. For other such experiments see Levinson 1996a.

5. In a workshop organized by Haviland and myself at the Linguistics Institute of Australia, Sydney, July 1992, the question of the distribution of such systems was addressed. It seems, from the information available, that most of the Australian languages make essential use of such systems with the exception of those in the Daly River area, where "upriver" and "downriver" notions may take their place. (Eva Schultze-Berndt, of our research group, has since investigated such a riverine system in Jaminjung, in the Timber Creek area of the Northern Territory.) Guugu Yimithirr may perhaps be somewhat unusual in the extent to which so-called topological and geometrical prepositionlike concepts are replaced by absolute direction expressions. The GY system of directionals is, however, not particularly elaborate as Australian systems go; for example, there is a much more complex set of morphological derivatives of cardinal direction roots in Warlbiri (Laughren 1978) or Kayardild (Evans 1995). Incidentally, I am grateful to the participants at that workshop for sharing their ideas and encouraging the line of work here described and for a subsequent correspondence with David Nash.

6. The information comes from Haviland n.d., where he gives thalbaarr as the alternate for jibaarr and nguwaar as the alternate for guwa. Although I found informants able to confirm this, they had no explanation. See also the following note.

7. The significance of the -rra suffix on the north and south terms, as opposed to the zero suffix on the west and east terms, is now opaque. The zero suffix on the latter might be interpreted as giving them some kind of conceptual priority, but there is no other evidence for this.

8. Many of these, but not all, are covered in Haviland 1993.

9. Of course, locative case plus place name may also suffice. It is the angular specification on the horizontal plane that requires use of cardinal directions. A number of further qualifications are in order here, but they must be left for another paper.

10. I may add by way of background that Hopevale was established as a mission in a large reservation, which covered part of the original territory of GY speaking peoples before the Palmer River goldrush of 1872 shattered their world. From 1886 to 1967 , the survivors from this and various other groups were collectively administered by Lutheran missionaries, who undermined many aspects of traditional culture, thereafter by a slowly evolving independent management (Haviland 1985; Haviland and Haviland 1980), recently in a complex context of land-right claims. Today the community numbers about 1,500 , but the pool of residents is in constant flux between this and other Queensland towns and reservations. As employment opportunities are low, most residents rely on social security payments, which together with school anchors most families to the Hopevale town center, but dispersed settlement and pastoral and hunting pursuits remain the favored way of 
life, now conducted with horses, guns, four-wheel-drive vehicles and outboardpowered dinghies to complement spear and woomera. Much traditional hunting and gathering knowledge persists, but most food comes from the central store. At the time of fieldwork, overland communication was still by dirt road, difficult in the wet season, but regular light airplane services were transforming travel.

11. The excellent grammatical and lexicographical work by Haviland describes the language largely as it was spoken by elderly speakers $\mathbf{2 0}$ years ago. It is still spoken that way, but by increasingly few people. There are extensive grammatical and lexical changes underway, in the direction, it seems, of simplification.

12. Work on acquisition of the language, and especially its spatial aspects, has been begun by Lourdes de León (de León n.d.[a]).

13. Large parts of the corpus, filmed or tape-recorded by J. B. Haviland since 1971 and by myself and Penelope Brown in 1982 and again in 1992, are deposited in the Australian Institute for Aboriginal and Torres Straits Islander Studies, Canberra, or are held at the Max Planck Institute for Psycholinguistics, Nijmegen, Netherlands.

14. Details of this "kit" of elicitation techniques are available from the Cognitive Anthropology Research Group, P.O. Box 310, Nijmegen, Netherlands.

15. The tasks have various sources of inspiration, especially Clark and WilkesGibbs 1986, von Stutterheim and Carroll 1989, and Weissenborn 1986, and were developed in the Max Planck Cognitive Anthropology Group jointly but with initial impetus by Lourdes de León (see de León n.d.[b]).

16. I regret now that I did not carry out the following simple experiment: $A$ tells $B$ how assemblage $X$ is, and $B$ is then asked to remake the assemblage the next day.

17. For speculations here, see the conclusions to the section "The Experiments: Discussion," below. John Haviland suggests to me that the possibility that the cardinal directions are reconstructed at the moment of speaking should be taken more seriously than this article suggests. Instead of extra memory requirements, GY speakers would then have extra inferential requirements (dead reckoning to the memorized location). It is not impossible that both mechanisms (memory of directions and inference of directions) are involved.

18. The ethnography of way-finding is, with the exception of studies of Oceanic navigation, extremely poor (see Levinson 1996b for review). Amazingly, even studies of hunter-gatherers usually omit any systematic survey of navigational techniques (cf. Widlok in press). The importance of such data to the understanding of prehistory should hardly need to be stressed.

19. Often the location pointed to had some angular breadth, for example, a mountain, island, or cape. In this case any gesture falling within the relevant arc was considered absolutely accurate, and errors were measured from outside the edges of the arc.

20. Comparable data from European subjects was collected by my colleague Eric Pederson, by asking Dutch amateur mycologists, foraging in semifamiliar woods, to point to local and distant locations. Their estimations were little better than random (see Levinson n.d.[b]). With larger samples and simpler tasks, Baker (1989) found statistically significant tendencies in the correct direction, but still less than half the British subjects judged locations to be in the correct $90^{\circ}$ quadrant. This material is reviewed in Levinson n.d.[b].

21. Lewis (1976) found accuracy vastly increased when pointing to sacred sites, and the same is probably true for locations of social and historical significance to Hopevale people.

22. I obtained this bush lore from two men who had worked as stockmen on stations outside the reservation, and it may well be station lore rather than aboriginal 
in origin. (For example, the star knowledge is suspect, given the lack of traditional names for constellations, but see Lewis 1976:274.)

23. Armed with a compass, I later checked out the locations. The questions, though, included "What direction did the TV in your room face?" which I was not always able to verify, but I am now quite prepared to believe!

24. This was the "Pear Film" produced by the Berkeley project for the cross-linguistic study of discourse, and I thank Wallace Chafe for permission to use it and Jack Dubois for a copy and the associated instructions.

25. I thank the architect of the Hopevale Rest Home for so disposing things, but that was luck; I thank the administrator of the home, Thelma McIvor, for permission to use the rooms, but that was kindness.

26. See Feinberg 1980:175 for extensive discussion of the application of the chi-square test to contingency tables with zero and low frequency cells.

27. This idea I think I owe to John Haviland in conversation, and certainly thanks is due to him for the ideas for some of the probes here described. Nick Evans (1995) also raises the subject in connection with the use of another Australian language, Kayardild.

28. Mythical stories are oriented, but given their connection to the landscape that is hardly surprising. One historical story described in Levinson $1987 \mathrm{~b}$ contains a sequence describing a fight in oriented fashion. There were no witnesses, and the protagonists either died or were transported to a penal settlement; so it is unlikely that the directions there, as opposed to the rest of the story, have historical veracity.

29. Alan Rumsey pointed this out to me. Incidentally, there are important questions about the impact of bilingualism and about Hopevale as a complex and fluid speech community where not all speakers control GY, which I am not equipped to pursue.

\section{References Cited}

\section{Baker, R. Robin}

1989 Human Navigation and Magnetoreception. Manchester, England: Manchester University Press.

Batschelet, Edward

1981 Circular Statistics in Biology. London: Academic Press.

Bowerman, Melissa

1996 The Origin of Children's Spatial Semantic Categories: Cognitive versus Linguistic Determinants. In Rethinking Linguistic Relativity. John Gumperz and Stephen C. Levinson, eds. Pp. 145-176. Cambridge: Cambridge University Press.

Bowerman, Melissa, and Soonja Choi

In press Shaping Meanings for Language: Universal and Language Specific in the Acquisition of Spatial Semantic Categories. In Language Acquisition and Conceptual Development. Melissa Bowerman and Stephen C. Levinson, eds. Cambridge University Press.

Brown, Penelope, and Stephen C. Levinson

1993 "Uphill" and "Downhill" in Tzeltal. Journal of Linguistic Anthropology 3(1):46-74.

Choi, Soonja, and Melissa Bowerman

1991 Learning to Express Motion Events in English and Korean: The Influence of Language-Specific Lexicalization Patterns. Cognition 41:83-121. 
Clark, Herbert

1973 Space, Time, Semantics and the Child. In Cognitive Development and the Acquisition of Language. Terrence E. Moore, ed. Pp. 27-64. New York: Academic Press.

Clark, Herbert, and Deanna Wilkes-Gibbs

1986 Referring as a Collaborative Process. Cognition 22:1-39.

de León, Lourdes

N.d.[a] The Development of Geocentric Location in Young Speakers of Guugu Yimithirr. Unpublished MS, Working paper \#33, Cognitive Anthropology Research Group, Nijmegen, Netherlands.

N.d.[b] Space Games in Tzotzil: Creating a Context for Spatial Reference. Unpublished MS, Working paper \#4, Cognitive Anthropology Research Group, Nijmegen, Netherlands.

Evans, Nicholas

1995 A Grammar of Kayardild. Berlin: Mouton Grammar Library.

Feinberg, Stephen E.

1980 The Analysis of Cross-Classified Categorial Data. Cambridge, MA: MIT Press.

Gumperz, John J., and Stephen C. Levinson, eds.

1996 Rethinking Linguistic Relativity. Cambridge: Cambridge University Press. Haviland, John

1979 Guugu Yimidhirr. In Handbook of Australian Languages. Robert M. W. Dixon and Barry J. Blake, eds. Vol.1.Pp. 27-182. Canberra: Australian National University Press.

1985 The Evolution of a Speech Community: Guugu Yimidhirr at Hopevale. Aboriginal History 9:170-204.

1993 Anchoring, Iconicity, and Orientation in Guugu Yimithirr Pointing Gestures. Journal of Linguistic Anthropology 3(1):3-45.

1996 Projections, Transpositions, and Relativity. In Rethinking Linguistic Relativity. John J. Gumperz and Stephen C. Levinson, eds. Pp. 269-323. Cambridge: Cambridge University Press.

N.d.[a] Complex Referential Gestures. Unpublished MS, Center for Advanced Study in the Behavioral Sciences, Palo Alto, CA.

N.d.[b] Guugu Yimithirr Vocabulary. Unpublished MS available in computer readable form from the AIATSIS Studies Electronic data archive no. 0011-0, Canberra, Australia.

Haviland, John, and Leslie K. Haviland

1980 How Much Food Will There Be in Heaven? Lutherans and Aborigines around Cooktown before 1990. Aboriginal History 4(2):118-149.

Johnson-Laird, Philip N.

1983 Mental Models: Towards a Cognitive Science of Language, Inference, and Consciousness. Cambridge: Cambridge University Press.

Johnston, Judith R., and Dan I. Slobin

1978 The Development of Locative Expressions in English, Italian, Serbo-Croatian and Turkish. Journal of Child Language 6:529-545.

Laughren, Mary

1978 Directional Terminology in Warlpiri. Working Papers in Language and Linguistics, 8. Launceston, Australia: Tasmanian College of Advanced Education.

Levelt, Willem J. M.

1989 Speaking. From Intention to Articulation. Cambridge, MA: MIT Press. 
Levinson, Stephen C.

1987a Pragmatics and the Grammar of Anaphora: A Partial Pragmatic Reduction of Binding and Control Phenomena. Journal of Linguistics 23:379-434.

1987b Minimization and Conversational Inference. In The Pragmatic Perspective. Jef Verschueren and Marcella Bertuccelli-Papi, eds. Pp. 61-129. Amsterdam: John Benjamins.

1996a Frames of Reference and Molyneux's Question: Cross-Linguistic Evidence. In Language and Space. Paul Bloom, Mary Peterson, Lyn Nadel and Merrill Garrett, eds. Pp. 109-169. Cambridge, MA: MIT Press.

1996b Language and space. Annual Review of Anthropology 25:353-382.

N.d.[a] Language and Cognition: The Cognitive Consequences of Spatial Description in Guugu Yimithirr. Unpublished MS, Working paper \#13, Cognitive Anthropology Research Group, Nijmegen, Netherlands.

N.d.[b] The Role of Language in Everyday Human Navigation. Unpublished MS, Working paper \#38, Cognitive Anthropology Research Group, Nijmegen, Netherlands.

N.d.[c] The Semantics/Pragmatics/Kinesics of Space in Guugu Yimidhirr. Unpublished MS, Bamberg, Germany.

Lewis, David

1976 Observations on Route Finding and Spatial Orientation among the Aboriginal Peoples of the Western Desert Region of Central Australia. Oceania 46(4):249-282.

Lucy, John

1992 Grammatical Categories and Cognition: A Case Study of the Linguistic Relativity Hypothesis. Cambridge: Cambridge University Press.

Miller, George, and Philip Johnson-Laird

1976 Language and Perception. Cambridge, MA: Harvard University Press.

Slobin, Dan I.

1991 Learning to Think for Speaking: Native Language, Cognition, and Rhetorical Style. Pragmatics 1:7-25.

1996 From "Thought and Language" to "Thinking for Speaking." In Rethinking Linguistic Relativity. John J. Gumperz and Stephen C. Levinson, eds. Pp. 70-96. Cambridge: Cambridge University Press.

Von Stutterheim, Christiane, and Mary Carroll

1989 Raumkonzepte in Produktionsprozesse. Unpublished MS.

Weissenborn, Jürgen

1986 Learning How to Become an Interlocutor. The Verbal Negotiation of Common Frames of Reference and Actions in Dyads of 7-14 Year Old Children. In Children's Worlds and Children's Language. Jenny Cook-Gumperz, William A. Corsaro, and Jürgen Streeck, eds. Pp. 377-404. Berlin: Mouton de Gruyter.

Widlok, Thomas

In press Orientation in the Wild: The Shared Cognition of Hai//6m Bushpeople. Journal of the Royal Anthropological Institute 3(2):317-332. 